\title{
El blog: un recurso didáctico que favorece la comprensión en los estudiantes sobre la formulación de compuestos inorgánicos ${ }^{1}$
}

\author{
Jennifer Julieth Lambraño López ${ }^{2}$
}

\begin{abstract}
Resumen
El presente proyecto deriva de la práctica pedagógica y didáctica realizada en el Colegio Champagnat de la ciudad de Bogotá, donde se contó con la participación de 30 estudiantes de grado décimo, con quienes se trabajó una serie de actividades diseñadas en el blog Químik Grado Décimo Colegio Champagnat, a partir del modelo de enseñanza para la comprensión (EPC) para el tópico generativo "Formulación de compuestos inorgánicos". La implementación de esta estrategia y los continuos seguimientos en los procesos de evaluación permiten evidenciar el favorecimiento de la comprensión en dicho tópico, siendo el uso del blog un recurso positivo para la enseñanza de la Química.
\end{abstract}

\section{Palabras clave}

Enseñanza para la comprensión, formulación de compuestos inorgánicos, práctica pedagógica y didáctica

\footnotetext{
Abstrac

This project stems from the pedagogical and didactic practice done in the Champagnat College of Bogotá, there, with the participation of 30 tenth graders, in which a series of activities designed blog was applied Químik Grado Décimo Colegio Champagnat on the basis of the model of Teaching for Understanding (EFC) for the generative topical formulation of inorganic compounds. The implemen-

1 Proyecto de Práctica Pedagógica y Didáctica II, realizado durante el segundo periodo de 2013 con la asesoría de la profesora Sandra Ximena Ibáñez, del Departamento de Química de la Universidad Pedagógica Nacional, y el acompañamiento de la profesora titular Laksmi Latorre Martínez, del Colegio Champagnat.

2 Estudiante de Práctica Pedagógica y Didáctica. Universidad Pedagógica Nacional. Correo electrónico: dqu_jlambrano515@pedagogica.edu.co.
} 
tation of this strategy and the continuous monitoring of assessment results allow concluding that the understanding of the topic in the students favored, with the use of a positive blog for chemistry teaching resource.

\section{Keywords}

Teaching for understanding, developing inorganic, education and teaching practice

\section{Introducción}

En la actualidad, las tecnologías de la información y la comunicación (TIC) pueden contribuir a los fines educativos contemplados en los currículos institucionales, que a su vez permiten desarrollar la capacidad creativa de estudiantes.

Tras la implementación del estudio exploratorio realizado en Práctica Pedagógica y Didáctica I, surgió el presente proyecto, con el cual se pretende mejorar la comprensión de los estudiantes de grado décimo del Colegio Champagnat en relación con el tópico "Formulación de compuestos inorgánicos". El trabajo tomó como referencia el modelo de enseñanza para la comprensión (EPC), y se hizo uso del blog Químik Grado Décimo Colegio Champagnat como recurso didáctico.

Castillo (2008) realizó un estudio sobre el aprendizaje de conceptos químicos desde el análisis fitoquímico del madroño, mediante la enseñanza para la comprensión, en la Institución de Educación del Distrito Codema J.M. (Bogotá, Colombia). Este diseño reconoció que las personas aprenden construyendo su propia comprensión durante procesos que involucran conocimientos previos y experiencias, siguiendo un círculo de aprendizaje.

Entre otros trabajos se destacan el de García (2013) y el de Jubert, Pogliani, Vallejo y Tocci (2011). Este último se llevó a cabo en la Universidad Nacional de La Plata (Argentina).

\section{Pregunta problema}

¿Cuáles indicadores para la comprensión pueden alcanzar los estudiantes de grado décimo en el estudio de la formulación de compuestos inorgánicos, haciendo uso del blog Químik Grado Décimo Colegio Champagnat?

\section{Objetivos}

\section{General}

Implementar actividades y dinámicas de comunicación en el blog Químik Grado Décimo Colegio Champagnat, que favorezcan la comprensión en los estudiantes sobre la formulación de compuestos inorgánicos.

\section{Específicos}

Diseñar actividades interactivas y lúdicas que promuevan la comprensión en los estudiantes sobre la formulación compuestos inorgánicos.

Evaluar la pertinencia de las actividades propuestas en el blog como medio para el desarrollo de la comprensión en los estudiantes.

\section{Marco teórico}

La enseñanza para la comprensión (EPC) implica para los profesores la posibilidad de reflexionar acerca de la práctica docente y su significación, y para los alumnos, la posibilidad de "despertar un interés reflexivo hacia las materias que están aprendiendo y ayudarlos a establecer relaciones entre su vida y la asignatura, entre los principios y la práctica, entre el pasado y el presente y entre el presente y el futuro" (Blyte, 1999, p. 36). 


\section{Cuatro preguntas acerca de} la enseñanza

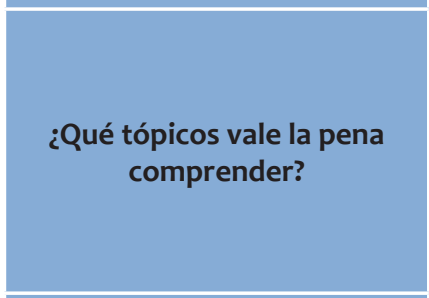

¿Qué aspectos de esos tópicos deben ser comprendidos?

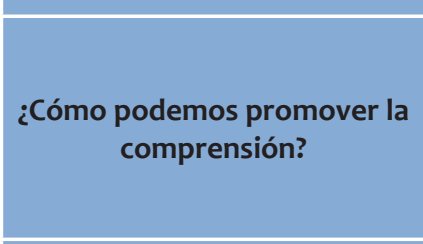

¿Cómo averiguar lo que comprenden los estudiantes?

\section{Aspectos de la enseñanza para la comprensión que abordan cada una de las preguntas}

Tópicos generativos: delinean la materia que los estudiantes investigarán por lo cual deben cumplir con las siguientes características:

- Ser centrales para un dominio o disciplina.

- Accesibles e interesantes para los alumnos.

- Interesantes para los docentes.

- Ricos en conexiones.

Metas de comprensión: afirman explícitamente lo que se espera que los alumnos lleguen a comprender; es decir, definen de manera más específica las ideas, procesos, relaciones o preguntas que los estudiantes comprenderán mejor por medio de su indagación. Estas deben ser explicitas y públicas, estar dispuestas en una estructura compleja y ser centrales para la materia.

Desempeños de Comprensión: actividades que desarrollan y a la vez demuestran la comprensión del estudiante en lo referente a las metas de comprensión; aquí encontramos tres categorías progresivas: etapa de exploración, investigación guiada y proyecto final de síntesis.

Evaluación diagnóstica continua de desempeños en relación con las metas de comprensión: proceso por el cual los estudiantes obtienen retroalimentación continua para sus desempeños de comprensión con el fin de mejorarlos.

Cuadro 1. Pilares de la enseñanza para la comprensión (Stone, 1999, pp. 15-22).

\section{Metodología}

La figura 1 muestra la estructura metodológica general utilizada para el desarrollo del proyecto.

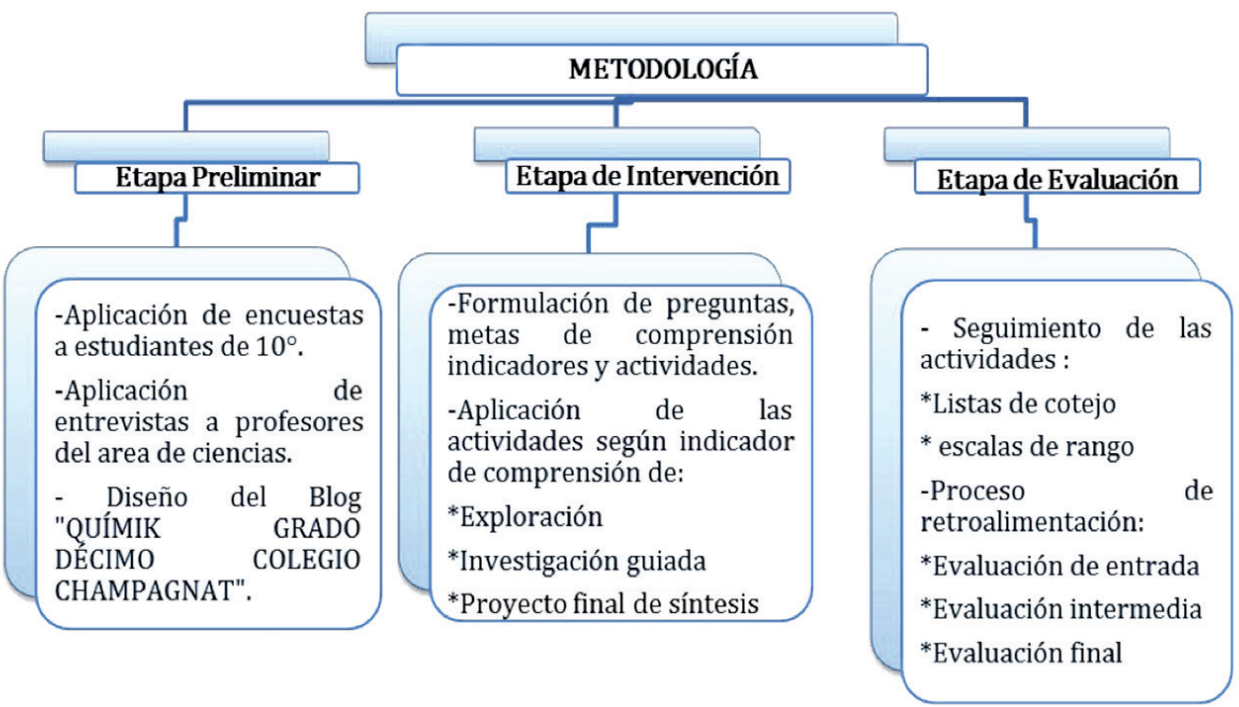

Figura 1. Estructura general del proyecto 


\section{Resultados y análisis}

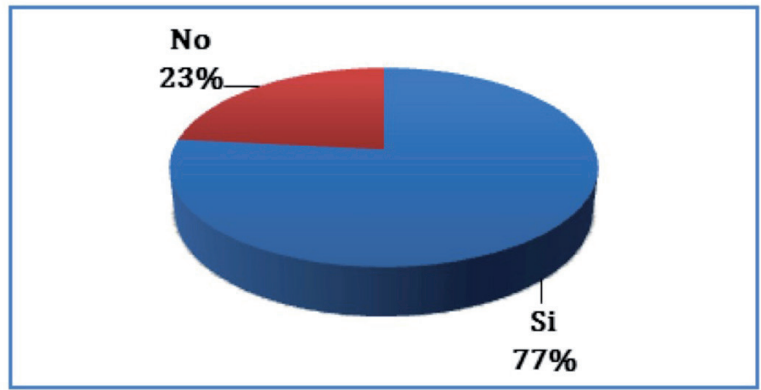

Fuente: Datos obtenidos en la escala de Rango

Gráfica 1. Porcentaje de estudiantes que alcanzaron el indicador: Reconoce que las bases se formulan a partir de óxidos básicos

Teniendo en cuenta los resultados obtenidos (anexo 1), en las actividades realizadas para dar cumplimiento a la meta de comprensión 1 (Diferenciar óxidos básicos de los óxidos ácidos), es evidente que $23 \%$ de los estudiantes (gráfica 1) aún no diferencia los óxidos básicos de los óxidos ácidos; esto se debe a que no han logrado entender que los óxidos básicos se forman de la combinación de un metal con el oxígeno y que los óxidos ácidos se forman a partir de un no metal y el oxígeno; no se reconoce que cada una de estas sustancias posee características distintas; es decir, que unas son de naturaleza básica y las otras de naturaleza ácida.

Por otro lado, $77 \%$ de los estudiantes (Gráfica N¹) comprende que los óxidos básicos al reaccionar con el agua permiten la obtención de hidróxidos o bases, lo cual progresivamente permite entender la diferenciación entre los dos tipos de óxidos.

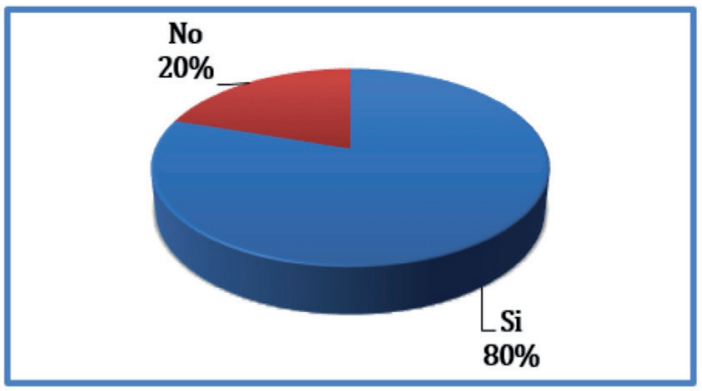

Fuente: Datos obtenidos en la escala de Rango

Gráfica $\mathbf{N}^{\circ}$ 2. Porcentaje de estudiantes que alcanzaron el indicador: "Nombra de manera adecuada cada compuesto, teniendo en cuenta las nomenclaturas"
Por otra parte, dar nombre a los compuestos inorgánicos a partir de la nomenclatura tradicional, Stock y IUPAC se muestra complejo en el $20 \%$ de los estudiantes (gráfica 2), esto debido a que no siguen de manera adecuada las instrucciones que cada una de estas nomenclaturas plantea, mientras que para la mayoría ha sido fácil relacionar a cada una de ellas.

Por ejemplo, a la pregunta "¿Cómo nombraría un compuesto inorgánico teniendo en cuenta las tres nomenclaturas?", algunos estudiantes respondieron:

Profe, simplemente se debe tener en cuenta que para la nomenclatura tradicional se utilizan los sufijos ico (mayor estado de oxidación) y oso (menor estado de oxidación); para la nomenclatura Stock se tiene en cuenta el número de oxidación del metal el cual se escribe entre paréntesis y en números romanos, y finalmente para la IUPAC 0 también conocida como sistemática se deben tener en cuenta los prefijos di, tri, tetra, etc. (Estudiante No. 9).

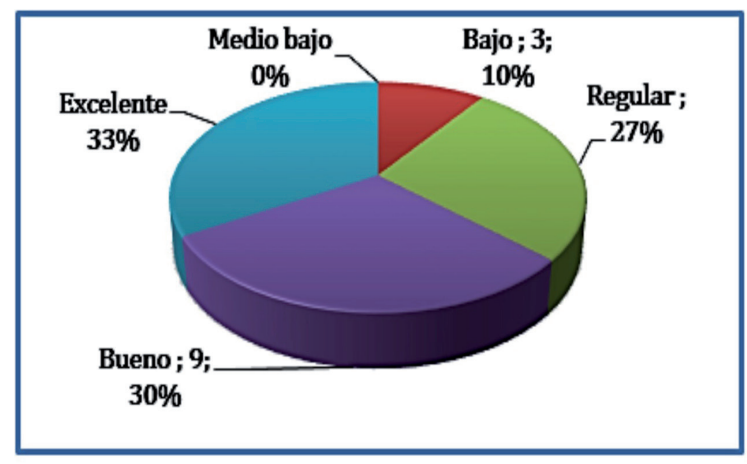

Fuente: Datos obtenidos en la escala de Rango

Gráfica $\mathbf{N}^{\circ}$ 3. Niveles alcanzados por los estudiantes para el indicador "Realizó los ejercicios propuestos en los interactivos de manera completa"

En la gráfica 3 se puede evidenciar que para el 63 $\%$ (suma de porcentajes: bueno y excelente) de los estudiantes el tema de formulación de compuestos inorgánicos es importante; esto se muestra en el desarrollo de las actividades propuestas en el blog y en clase, lo cual permite analizar cómo incide la interactividad para captar la atención de los estudiantes y generar en ellos el gusto por la química (Galvis, Hernández, Mendoza y Marenco, 1999). 


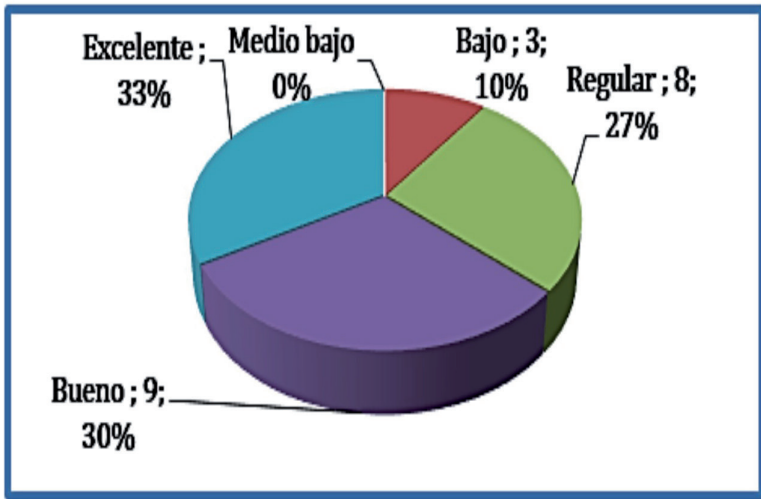

Fuente: Datos obtenidos en la escala de Rango

Gráfica $\mathrm{N}^{\circ} 4$. Niveles alcanzados por los estudiantes para el indicador "Entregó la solución de los ejercicios desde la formulación del óxido hasta el compuesto correspondiente"

En la gráfica 4 se observa que para $90 \%$ (suma de porcentajes: regular, bueno y excelente) de los estudiantes, es fundamental formular los compuestos inorgánicos desde su óxido correspondiente, ya que de esta manera se logra un estudio positivo para el aprendizaje de esta temática; también, para la población trabajada, es importante la nomenclatura de cada compuesto, pues esta resalta la identidad y propiedad respectiva de cada sustancia, lo cual se reconoce en los resultados expresados en la gráfica 2.

Ahora bien, mediante el foro virtual se puede identificar cómo los estudiantes logran argumentar a la actividad: "Cada vez que mamá hace aseo, suele utilizar blanqueador para desinfectar los pisos y paredes, y también para blanquear la ropa blanca con el fin de que esta conserve su color... ¿A qué compuesto inorgánico se le debe tan importante función?". Al respecto se dieron respuestas como:

Esta importante función se le debe al hipoclorito de sodio ( $\mathrm{NaClO})$, el cual al contener el elemento químico cloro en estado de oxidación +1 actúa como un oxidante fuerte y económico. Debido a esta característica se utiliza como desinfectante, además destruye muchos colorantes por lo que actúa como blanqueador" (Estudiante No. 21).

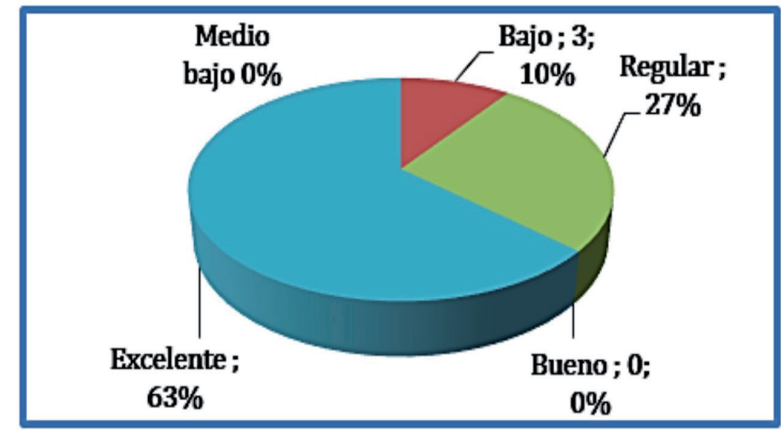

Fuente: Datos obtenidos en la escala de Rango

Gráfica $\mathbf{N}^{\circ}$ 5. Niveles alcanzados por los estudiantes para el indicador "Participó en el foro Formulación Orgánica, argumentando adecuadamente la importancia del ácido clorhídrico en el uso doméstico, sus propiedades desinfectantes y por qué de ellas"

Lo anterior, refleja la importancia de realizar actividades que involucren situaciones de la vida cotidiana y desde las TIC favorecer la comprensión en los estudiantes, lo cual se debe a las múltiples características y oportunidades de interactividad que ofrece este recurso tecnológico. En la gráfica 5, se observa la participación del $90 \%$ (suma de porcentajes: regular y excelente) de los estudiantes en el foro, en el cual exponen desde diferentes formas de argumentación las características del compuesto inorgánico hipoclorito de sodio y sus múltiples funcionalidades domésticas e industriales.

Por lo anterior, se resalta que el modelo de enseñanza para la comprensión trabajado desde las tic fomenta y favorece de manera positiva la comprensión de la formulación de compuestos inorgánicos en los estudiantes; esta afirmación se puede analizar con los resultados de la lista de cotejo para las actividades de la meta de comprensión No. 3 y No. 4, donde es evidente que la mayoría de la población estudiada formula y nombra de manera adecuada los compuestos inorgánicos, diferencia entre un ácido orgánico e inorgánico y reconoce la importancia de estos en la industria y la cotidianidad; igualmente diferencia entre un oxácido y un hidrácido cuando expone que los oxácidos son aquellos que en su estructura tienen al elemento oxígeno y los hidrácidos carecen de este. También, gracias a los seminarios planteados por grupos de trabajo se logró que los estudiantes reconocieran la importancia de algunos compuestos inorgánicos presentes en el me- 
tabolismo del cuerpo como: el funcionamiento del ácido clorhídrico ( $\mathrm{HCL}$ ) en el estómago y las consecuencias que traería su rendimiento normal; las sales inorgánicas excretadas por el sistema urinario y los problemas a nivel inmunológico derivados del exceso de estas en el organismo, entre otros. (Yankovie, 2007, p. 65).

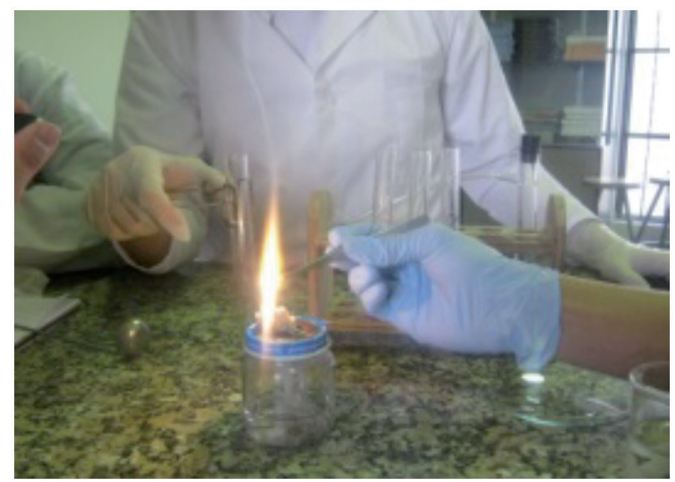

Fotografía 1. Oxidación del magnesio.

Por último, se evidencia que mediante la práctica de laboratorio realizada (fotografía 1), los estudiantes reconocieron las funciones y reacciones inorgánicas que se evidencian en la naturaleza, en la cotidianidad y en la industria; esto se logró al oxidar una puntilla, donde se explica la oxidación de los metales cuando se encuentran en presencia del oxígeno del ambiente o la corrosión de los mismos como suele conocerse.

\section{Conclusiones}

El blog Químik Grado Décimo Colegio Champagnat es un recurso didáctico que permite la interactividad entre los estudiantes y el mundo cibernético, favoreciendo la comprensión del tópico "Formulación de compuestos inorgánicos". Teniendo en cuenta esto y a partir de los resultados en cada una de las actividades propuestas en el blog, es evidente que mediante el modelo EPC los estudiantes alcanzaron los indicadores de comprensión sobre la formulación de compuestos inorgánicos, partiendo de actividades exploratorias, pasando por actividades de investigación guiada y llegando a las actividades de proyecto final y síntesis; en esta etapa los alumnos han llegado a un nivel superior de la comprensión.

La evaluación de este proceso de comprensión se dio gracias al uso de instrumentos como la lista de cotejo y la escala de rango donde se pudo analizar cada desempeño alcanzado en las etapas inicial, intermedia y final.

No solamente las actividades interactivas favorecen la comprensión en los estudiantes; también las lúdicas y experimentales, puesto que ponen de manifiesto las habilidades de los estudiantes en la experiencia y en el trabajo grupal; esto se pudo observar, por ejemplo, en la práctica de laboratorio y en la realización de los seminarios donde los alumnos expusieron sus argumentos sobre la importancia y funcionamiento de los compuestos inorgánicos en el metabolismo del cuerpo humano.

\section{Bibliografía}

Blyte, T. (1999). La enseñanza para la comprensión. Buenos Aires. Paidós.

Castillo, H. (2008). Aprendizaje de conceptos químicos desde el análisis fitoquímico del madroño aplicando enseñanza para la comprensión. Recuperado el 6 de julio de 2012 de: http://portalweb.ucatolica. edu.co/easyWeb2/files/44_200_v2n3castillo.pdf

Galvis, A.; Hernández, A.; Mendoza, P. y Marenco, E. (1999). Ambientes virtuales del aprendizaje: enseñanzas del proyecto OLLyT. Informática Educativa, Uniandes-LIDIE. Vol. 12, No, 2. pág. 275-277.

García, J. (2013). Equilibrio ácido base y espectrofotometría molecular en el espectro visible. Propuesta de enseñanza desde el enfoque enseñanza para la comprensión "EPC". Bogotá. Revista del Sistema de Práctica Pedagógica y Didáctica, 51. pág. 1-7.

Jubert, A.; Pogliani, C.; Vallejo, A. y Tocci, A. (2011). Enseñanza para la Comprensión en un curso de química a distancia de nivel básico universitario. El blog como herramienta de trabajo Avances en ciencia y tecnología. Buenos Aires: Universidad Nacional de La Plata.

Stone, M. (1999). La enseñanza para la comprensión. Vinculación entre la investigación y la práctica. Buenos Aires: Paidós.

Yankovie, B. (2007). El genoma humano al alcance de todos. Santiago de Chile: Editorial ABCiencia RIL. 


\section{Anexos}

\section{ANEXO $\mathrm{N}^{\circ} 1$}

Lista de cotejo para las actividades de la meta de comprensión $\mathrm{N}^{\circ} 1$ (si/no)

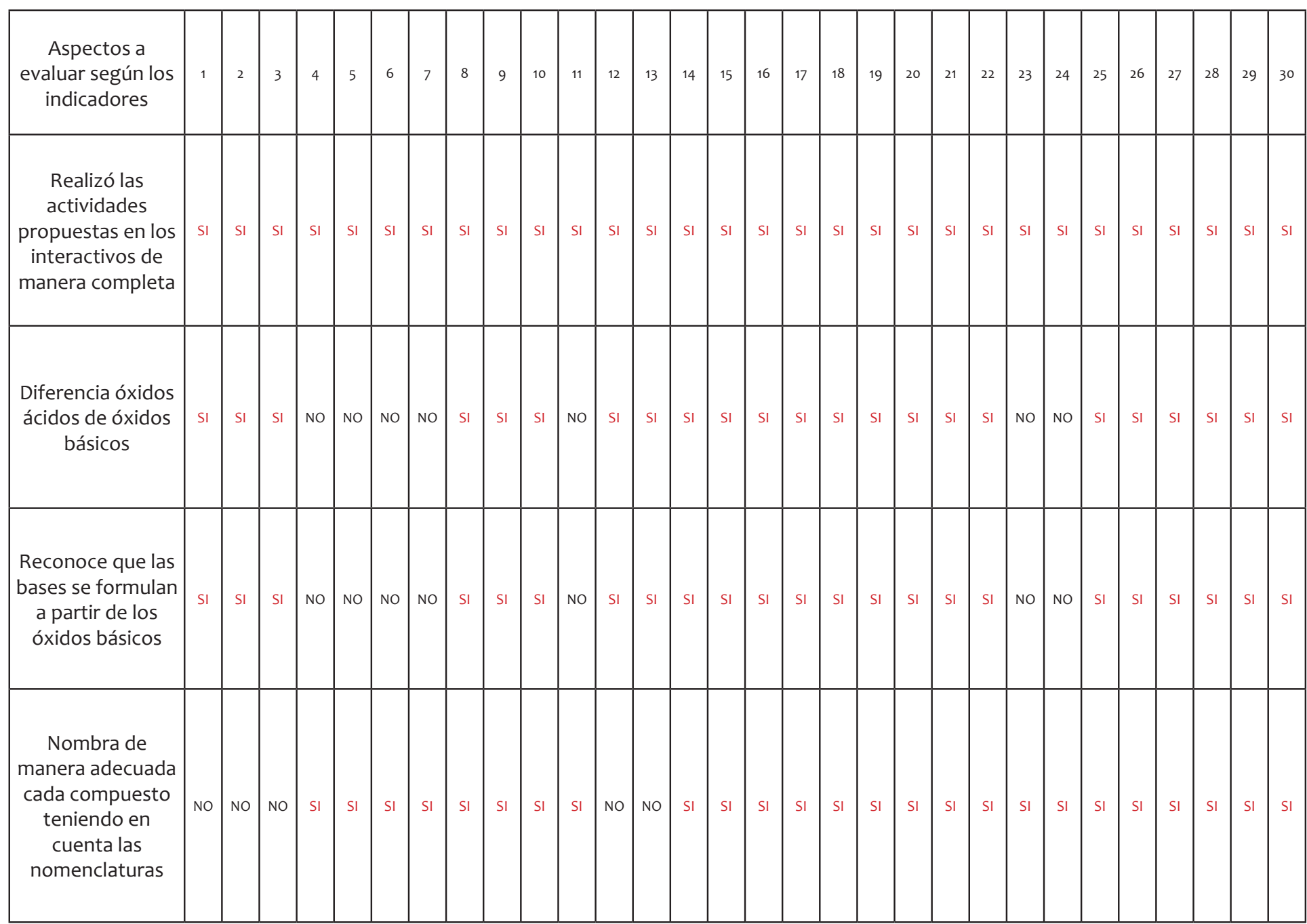

